\title{
Design for the Methodology of Plant Leaf Disease Detection using Image Processing
}

\author{
Prabhjeet kaur $^{1}$ and DR. Sanjay Singla ${ }^{2}$
}

Abstract - Indian Economy is highly dependent on productivity of agricultural crops/plants. So, there is the need to provide extreme attention to take care of plants/crops. Diseases are the natural factor that can cause some serious effects on plants which ultimately reduces productivity, quality and quantity of products. Manual detection of plant diseases just increases the human efforts as it is not easy to check each individual plant. Also the manual detection is not appropriate method. In this paper, we have presented the design of an autonomous approach for the detection of plant leaf diseases. The proposed method covers the steps of image processing technique. These image processing steps are image acquisition in RGB color value, image preprocessing using histogram equalization, image segmentation using k-means clustering, feature extraction with texture statistics, image classification using Support Vector Machine and final optimization using Particle Swarm Optimization method. So, overall design for plant leaf disease detection is presented here.

Keywords - Plant Leaf Diseases Detection, Particle Swarm Optimization, Image Processing, Support Vector Machine

\section{INTRODUCTION}

There is the existence of a variety of plants on this earth surface that plays enormous role in human life [1]. As the fear of global warming increases due to reduction of plants on the earth surface, human has taken some important steps to save plants. In the ancient years, it was not easy to detect the plant diseases on time. But in this computing era, researchers have structured some efficient approaches and algorithms that can be used to detect plant diseases on time with leas man labor. In computing technology, digital image processing rapidly developed that it can be used for various real life applications. Image processing involves the steps of image acquisition, preprocessing, segmentation, feature extraction, classification and optimization [2]. Various authors have used different methods for the detection of plant leaf diseases.

Khirade et al. [3] have discussed some segmentation and feature extraction algorithm that can be used for the detection of plant diseases by using the images of their leaves. Rothe et al. [4] have proposed pattern recognition techniques for the detection and classification of cotton leaf diseases of Alternarnia, Myrothecium and Bacterial Blight.

Kaur and Kang [5] have proposed an enhanced neural network based support vector machine concept for the disease detection and disease affected portion in plant leafs.

Dandawate and Kokare [6] have used support vector machine concept for the detection and classification of soybean plants as diseased or healthy species. Authors have used the SIFT approach that automatically recognizes plant species by their leaf shape.

\footnotetext{
${ }^{1}$ Computer Science Engineering IET Bhaddal Technical Campus City: Rupnagar, Country: India

${ }^{2}$ Computer Science Engineering IET Bhaddal Technical Campus City: Rupnagar, Country: India
} 
Gavhale et al. [7] have performed the process of image processing for detection of unhealthy region of citrus leaf.

Kutty et al. [8] has used the neural network based system to classify the watermelon leaf diseases of Downey Mildew and Anthracnose. This classification is based on the color feature extraction from RGB color model.In this paper, we are using image processing technique for the detection of plant leaf diseases. Dataset of plant leaf affected from the diseases of Cercospora Leaf Spot, Bacterial Blight, Anthracnose and Alternaria Alternata are considered [3]. Here, image acquisition is performed by considering RGB colour distance affected leaf image. Image preprocessing is performed to enhance the image quality using histogram equalization. Image feature extraction is performed to extract the features of leaf disease symptoms. Image classification is performed using support vector machine and finally particle swarm optimization is performed for the optimization of concept. Other sections of the paper are described as: Section II presents the plant leaf disease sensing methods, Section III explains the design of proposed methodology with its work flow and Section IV concludes the paper.

\section{PLANT DISEASE SENSING METHODS}

There are various disease sensing methods like remote sensing, Thermograph Techniques, Laser Sensing, Visible Spectroscopy, Light Reflectance [4]. These methods are discussed below.

\section{A. Remote Sensing}

Remote sensing technology was used in agriculture for yield crop estimation. It depends on the spatial resolution of the digital image and it is affected in growing crops estimation. This method is more robust because it's established the relation between yield monitor data and remotely sensed images.

\section{B. Thermograph Techniques}

Thermograph techniques are depending upon the biomass of the fruit or disease. This technique refers to identifying and classifying between fruit and shrubs and trees. Thermography techniques convert the visible image to invisible radiation pattern and it has successfully adopted in agriculture. Various researches used thermographs to recognitions of fruits. The temperature of fruit and leaves are also helpful to differentiate by using thermography

\section{Laser Sensing}

Laser sensors are used for fruit recognitions technique. Image processing and laser based application systems are used for navigating a tractor through the alleyway of a citrus grove. Laser sensor gives the $3 \mathrm{D}$ information on agriculture crop and forage swaths, together with expert system which can identify the these features and overcome interference caused by sensible amounts of airborne dust.

\section{Visible Spectroscopy}

Visible spectroscopy techniques applied in soil testing and characterization. There arc various methods like NIR reflectance spectroscopy, Raman spectroscopy, VIS, VV, etc. which are applied in agriculture sector.

\section{E. Light Reflectance}


Light reflect work on law of reflection, and the result shows the reflection occurs off a curved surface or off a flat surface.

Light always never passes through the virtual actual image location it only appears to investigate as though the light is emanating from the virtual image location. Light reflectance is used for disease classification. Each disease has own fundamental color properties and light reflectance works on the bases of wave length and spectrum. The result shows that each disease has own bandwidth, wave length and spectrum which help to classify the disease.

\section{RESEARCH METHODOLOGY}

The proposed concept is conducted in MATLAB 8.3.0.532 with Windows 7 based system having $6 \mathrm{~GB}$ of RAM, 1TB of HDD, Intel(R) Core(TM) i5 CPU. Expert dataset is considered in image form of leaves that are affected with the diseases of Cercospora Leaf Spot, Bacterial Blight, Anthracnose and Alternaria Alternata. Here, we have presented the process of plant leaf disease detection using image processing. In this process, we have considered the disease affected leaf as input and evaluated the type of leaf disease so that plant can be cured further from that disease. Steps for the research methodology are described below with flowchart as shown in figure 1.

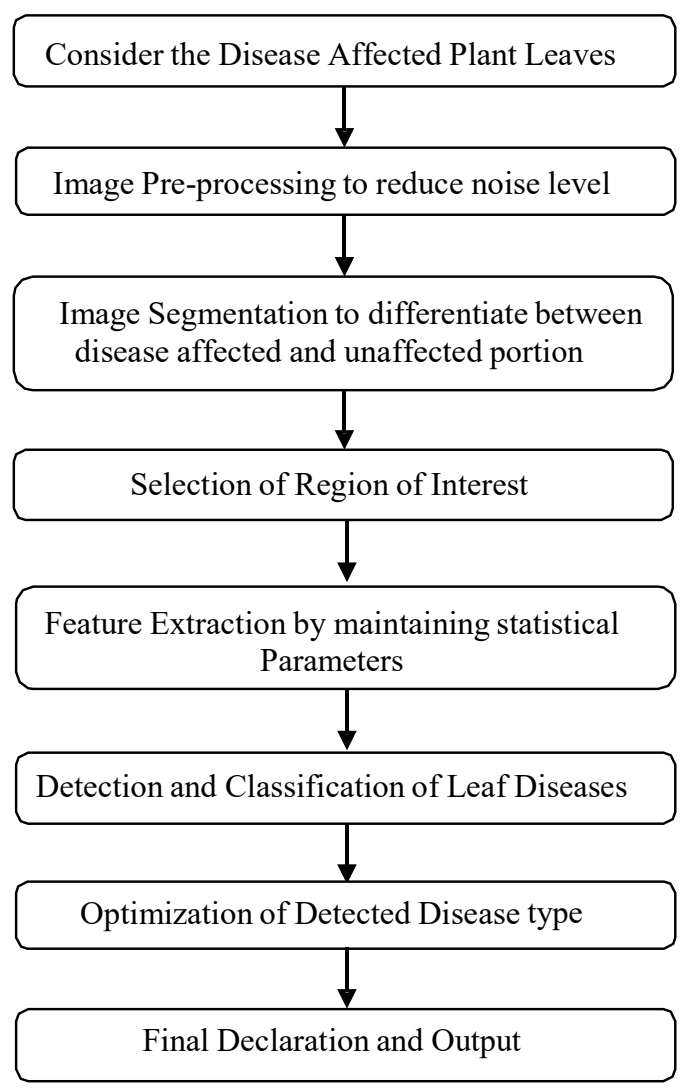

\section{Figure 1: Flowchart for the Proposed Concept}

The explanation for the work flow of proposed concept is described below.

Step 1: Consider the Disease Affected Plant Leaves.

Step 2: Pre-process the Image using histogram equalization method to reduce noise value in considered leaf image. 
Step 3: Perform Image Segmentation using K-means Clustering to cluster the image into leaf affected portion and unaffected one.

Step 4: Select the disease affected Region of Interest from the Segmented Image.

Step 5: Perform Feature Extraction by maintaining statistical Parameters of Skewness, Standard_Deviation, Homogeneity, Contrast, Smoothness, Correlation, Kurtosis, Energy, Entropy, Mean, Variance, RMS, and IDM.

Step 6: Apply Support Vector Machine for the Detection \& Classification of disease type.

Step 7: Apply Particle Swarm Optimization for the optimization of results.

Step 8: Declare the Disease type and evaluate the percentage of disease affected portion.

\section{CONCLUSIONS}

The detection and classification of leaf diseases accurately is the key to prevent the agriculture loss. There are a list of methods and classifiers to detect plant diseases. But in this research work, we are using image processing technique for the detection of plant leaf diseases. A framework for the design of plant leaf disease detection is presented her with its work flow. Dataset for the proposed concept is prepared with the plant leaf suffered with diseases Cercospora Leaf Spot, Bacterial Blight, Anthracnose and Alternaria Alternata. We have also analysed the disease affected portion of leaf. As per the designed framework, we can say that proposed concept of image processing is efficient enough to detect the plant leaf diseases.

\section{REFERENCES}

[1]. Vitousek, Peter M., Harold A. Mooney, Jane Lubchenco, and Jerry M. Melillo. "Human domination of Earth's ecosystems." Science 277, no. 5325 (1997): 494-499.

[2]. Leondes, Cornelius T. Image processing and pattern recognition. Vol. 5. Elsevier, 1998.

[3]. Quinn, B. L. P., J. de Vos, M. Fernandes-Whaley, C.Roos, H. Bouwman, H. Kylin, R. Pieters, and J. van den Berg. "Pesticides in the modern world-Pesticides use and management, ed. M. Stoytcheva." (2011).

[4]. Mahlein, Anne-Katrin, Erich-Christian Oerke, Ulrike Steiner, and Heinz-Wilhelm Dehne. "Recent advances in sensing plant diseases for precision crop protection." European Journal of Plant Pathology 133, no. 1 (2012): 197-209.

[5]. Khirade, Sachin D., and A. B. Patil. "Plant Disease Detection Using Image Processing." In Computing Communication Control and Automation (ICCUBEA), 2015 International Conference on, pp. 768-771. IEEE,2015.

[6]. Rothe, P. R., and R. V. Kshirsagar. "Cotton leaf disease identification using pattern recognition techniques." In Pervasive Computing (ICPC), 2015 International Conference on, pp. 1-6. IEEE, 2015.

[7]. Kaur, Rajleen, and Sandeep Singh Kang. "An enhancement in classifier support vector machine to improve plant disease detection." In MOOCs, Innovation and Technology in Education (MITE), 2015 IEEE 3rd International Conference on, pp. 135-140. IEEE, 2015.

[8]. Dandawate, Yogesh, and Radha Kokare. "An automated approach for classification of plant diseases towards development of futuristic Decision Support System in Indian perspective." In Advances in Computing, Communications and Informatics (ICACCI), 2015 International Conference on, pp. 794-799. IEEE, 2015. 
[9]. Gavhale, Kiran R., Ujwalla Gawande, and Kamal O.Hajari. "Unhealthy region of citrus leaf detection using image processing techniques." In Convergence of Technology (I2CT), 2014 International Conference for, pp. 1-6. IEEE, 2014.

[10]. Kutty, Suhaili Beeran, Noor Ezan Abdullah, Habibah Hashim, and Aida Sulinda. "Classification of watermelon leaf diseases using neural network analysis." In Business Engineering and Industrial Applications Colloquium (BEIAC), 2013 IEEE, pp.459-464. IEEE, 2013. 